\title{
A study of machine learning methods for detecting user interest during web sessions
}

\author{
Alípio M. Jorge \\ LIAAD - INESC TEC, DCC - \\ FCUP, Universidade do Porto, \\ Portugal \\ amjorge@fc.up.pt \\ Sarabjot S. Anand \\ Algorithmic Insight, India \\ ssanand@a-insight.com
}

\author{
José Paulo Leal \\ CRACS - INESC TEC, DCC - \\ FCUP, Universidade do Porto, \\ Portugal \\ zp@dcc.fc.up.pt \\ Hugo Dias \\ DCC - FCUP, Universidade do \\ Porto, Portugal
}

\begin{abstract}
The ability to have an automated real time detection of user interest during a web session is very appealing and can be very useful for a number of web intelligence applications. Low level interaction events associated with user interest manifestations form the basis of user interest models. However such data sets present a number of challenges from a machine learning perspective, including the level of noise in the data and class imbalance (given that the majority of content will not be of interest to a user). In this paper we evaluate a large number of machine learning techniques aimed at learning from class imbalanced data using two data sets collected from a real user study. We use the AUC, recall, precision and model complexity to compare the relative merits of these techniques and conclude that useful models with AUC above 0.8 can be obtained using a mix of sampling and cost based methods. Ensemble models can provide further accuracy but make deployment more complex.
\end{abstract}

\section{Keywords}

modeling; user interaction; user interest; machine learning; imbalanced classification

\section{INTRODUCTION}

The automatic detection of user interest while browsing is a very appealing ability of a web client. The browser may not only detect when the user is interested but also in what region of the page (where) the user is interested in. This opens up to many possible applications such as triggering recommendations when the user seems to be interested in a particular portion of text or image. The observation of the user behavior is based on low level client side interaction events with the mouse and keyboard. The browser keeps

Permission to make digital or hard copies of all or part of this work for personal or classroom use is granted without fee provided that copies are not made or distributed for profit or commercial advantage and that copies bear this notice and the full citation on the first page. To copy otherwise, to republish, to post on servers or to redistribute to lists, requires prior specific permission and/or a fee.

IDEAS14 July 7-9, Porto [Portugal]

Editor: Bipin C. DESAI

Copyright 2014 ACM 978-1-4503-1976-8/12/06 ...\$10.00. collecting these events as the user interacts. Such a stream is fed to a classification model, embedded in the code of the web page, which decides whether the user is showing interest or not. The result of the model can then be used on the client side or transmitted to a remote server for a more demanding response.

In our approach we use machine learning for obtaining the classification models automatically. Detecting user interest is modeled as a binary classification problem: interested/not interested. As expected, the proportion of classes is very imbalanced, given that interest events are relatively rare. Most of the time the user is simply browsing or is in an idle state. Therefore, one important challenge is how to robustly deal with the class imbalance. Other challenges, not addressed in this paper, are: how to collect the training data and how to pre-process the data in order to favor prediction.

In this paper we study the applicability of a large number of machine learning techniques for addressing class imbalance bearing in mind two aims. One is to have models that can detect a good number of interest situations with a proportion of false alarms as low as possible and the other is to obtain models that are lightly embeddable in the page and can run on the client side. The methods are evaluated on two data sets collected by us from real interaction data. We use 10-fold cross-validation and estimate the performance of the models using metrics such as the Area Under the Curve (AUC) [1], recall, precision and model size. From the empirical results we can rank the methods to be used for the purpose of detecting user's interest.

\section{MODELING USER INTERACTION}

As soon as web browsers and hypertext became popular researchers started trying to model interaction behavior. Letizia, for example, was a browser agent proposed by Lieberman [2]. It observed the browsing behavior of a particular user and learned to anticipate which pages would be of interest. It recorded actions on the document such as saving or hyperlink clicking and scored words in it. These were used to recommend pages containing highly scored words to be followed next. Letizia was implemented as what would nowadays be an applet.

Others have also exploited the recordable interaction provided by the new media. Claypool et al. [3] would see some 
human identified patterns of activity as implicit interest indicators. The user would provide, without noticing, information about his/her interest on pages solely by browsing. Not explicitly asking the user's opinion is also called unobtrusive observation by Goecks and Shavlik [4] who built a browser agent that collected interaction data and trained a neural network to predict the amount of interaction activity given the words of a page. This model can be used to automatically look for pages of interest to the user. These approaches for modeling user behavior do not rely on the traditional server-side clickstream data, but rather follow the philosophy of the curious browser [3] by collecting client-side data [5], also called subsymbolic user-behavior [6].

Browsing models may also attempt to determine, at any given instant a browsing state. Syeda-Mahmood and Ponceleon [7] have proposed 10 different states for users browsing videos, such as: curious, aimless browse, found something interesting, undetermined, etc. These authors have also exploited the interdependence of subsequent states using Hidden Markov Models.

Most of the above mentioned works studied or modeled user's interest on whole web pages or documents. Hijikata [8] tried to improve the user interest model on pages/words by focusing on parts of the browsed pages. These relevant parts were identified through specific interaction events such as text selection, link clicking and pointing, moving the mouse over the text while reading, etc. Although some of these actions are not necessary for browsing, they were unconsciously performed by users and carried interesting information. Hauger and Velsen [5] collected client-side interaction events for trying to identify which parts of a page had been read. They organized a controlled experiment with 53 users who had to fill in a questionnaire saying which parts of the page they actually read. This data was then used to train machine learning classification methods. Yang et al. [9] used a similar approach on small screen devices for identifying what they call interest blocks. Later, Hauger et al. proposed the use of eye-tracking, in addition to interaction data, and concluded that interactive behavior can be used to approximately determine gaze position [10].

\subsection{Our approach}

This paper is part of a larger endeavour for designing an end-to-end process for modeling user interest and deploying such models so that they can be used in real time. The process has two phases: learning and deployment. In the first phase we collect data and obtain a model. In the second we embed the model in the page and use it.

The learning phase has the following steps:

1. Select a web page as target;

2. Define an area of interest;

3. Define a question whose answer is in the defined area of interest;

4. Invite users to open the page and answer the question (as in a quiz) - users are not given any other information;

5. Collect user client-side interaction data (mouse and keyboard) during the quiz sessions;

6. Associate each interaction event (e.g. click or mousemove) to an $x, y$ location in the page, if possible;
7. Label each event location as in the area of interest ("INT") or outside of it ("BROWSE"):

8. Aggregate events in time contiguous segments;

9. Aggregate labels in the same segment for obtain a segment label;

10. Setup a classification data set from segments;

11. Build a classification model from the data set.

After learning we deploy the model embedded in the page.

1. Embed the model in the page as JavaScript;

2. Aggregate user interaction events on the client side as a sliding active segment;

3. Invoke the model upon every event using the active segment and obtain an interest label;

4. Use the interest label for recommendation or for collecting high level interaction events.

We currently have a complete solution for the whole process [11] but many parts of the process present interesting challenges. For the first steps of the learning phase we have setup user quizzes that were publicized in social networks and through email. As a result we had a very relevant number of users providing data without knowing exactly what it was for, which minimized biased behavior. In particular, we mirrored 2 relatively long web pages: one from Wikipedia on the Olympic Committee and the other from facebook on Soccer. For each of them we proposed a question. Upon entering the page the user would read the question in a popwindow, minimize the pop-up, look for the answer in the page (text find was not particularly useful since we avoided searchable words in the question), reopening the pop-up, and choose the right option as an answer. Fig 1 illustrates one of the quizzes.

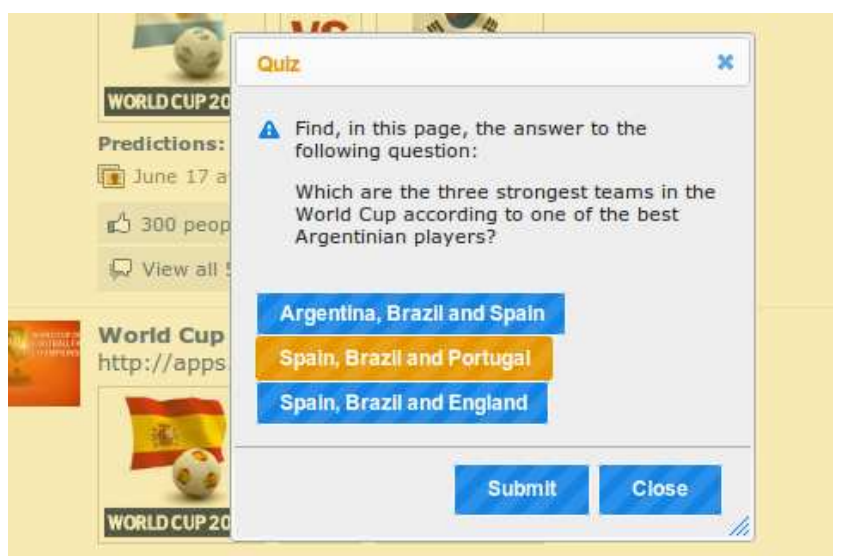

Figure 1: Screenshot of one of the pop-up quizzes as it appears to the user.

From the sessions generated on each of the two web page we have generated a data set. One will be referred to as the Olympic data set and the other as the Soccer data set. 
The aggregation referred in step 8 starts by segmenting the event stream in chunks of 5 seconds. Then the frequency of each event is counted (each frequency is an attribute of the data set). Finally the maximum range of the $x$ and $y$ coordinates, in the segment, related to event locations give rise to other two attributes. The label of a segmetn is INT if any event in the segment is labeled with INT, otherwise it is BROWSE. Tabel 1 describes the resulting data sets.

Table 1: The two resulting data sets.

\begin{tabular}{|l|r|r|r|r|r|}
\hline Data set & Size & \#BROW & \#INT & \%BROW & \%INT \\
\hline \hline Olympic & 1994 & 1789 & 205 & $90 \%$ & $10 \%$ \\
Soccer & 1610 & 1564 & 46 & $97 \%$ & $3 \%$ \\
\hline
\end{tabular}

In this paper we specifically focus on step 11 of the learning phase (building a classification model). Our purpose here is to study robust machine learning methods that can cope with the generated data. One very distinct feature of this data is its class imbalance. The number of segments where the user is going to show interest is relatively low (10\% and $3 \%$ in our experiments). This requires the use of algorithms for imbalanced data as well as an adequate evaluation methodology. In the next section we describe the techniques used in our study.

\section{IMBALANCED CLASSIFICATION}

Class Imbalance is typically addressed by data sampling. The simplest approach is to randomly undersample the majority class or randomly oversample (with replacement) the minority class. The amount of under(over)sampling depends on the domain being modelled and often does not simply translate to the generation of a uniformly distributed class label. Hence, Chawla et al. [12] proposed the use of crossvalidation to determine the optimal amount of sampling.

As opposed to simple under(over)sampling, more "intelligent" sampling approaches have also been proposed. The most significant such oversampling method is Synthetic Minority Over-sampling TEchnique (SMOTE) [13]. The removal of redundant negative instances and tomek links was proposed as an alternative to undersampling [14]. In SMOTE, synthetic examples are generated from each positive training instance along the (hyper-)plane joining the positive instance to one of its $k$ nearest positive instance neighbours. In SMOTE, synthetic examples are generated from each positive training instance, $t_{i+}$, (the seed instance) as follows. First the $k$ nearest neighbours, $n_{i t}$ 's of $t_{i+}$ are retrieved. Next $r$ of these nearest neighbours are chosen through sampling by replacement, where $r$ is the number of synthetic examples that each of the positive training instances will contribute to the new oversampled training data set. For example, if $300 \%$ oversampling is to be carried out then $\mathrm{r}$ $=3$. The synthetic data instance $s_{i j}$ is then generated as $\vec{s}_{i j}=\vec{t}_{i+}+q \cdot\left(\vec{n}_{i j}-\vec{t}_{i+}\right)$ where $q$ is a random number between 0 and 1 . Note that Oversampling with replacement is a special case of SMOTE where $q$ is set to 0 . Kubat and Matwin [14] proposed a method for undersampling the negative examples by removing redundant instances and tomek links as opposed to random undersampling. The new training set is seeded with all positive instances and a randomly selected negative instance. Using the seed instances as the model, the remaining negative instances are classified using the 1-Nearest Neighbour algorithm and misclassified neg- ative instances are added to the new training set. Finally those negative instances with a positive instance as the nearest neighbour (referred to as tomek links) are removed.

Both, undersampling and oversampling have drawbacks. First, undersampling leads to loss of information as negative instances not used in learning could impact model performance on unseen examples. On the other hand, oversampling can result in over-fitting and an increase in learning time as the training data can increase substantially in size as a result.

More recently, repetitive sampling-based ensemble models have also been proposed. Seiffert et al. applied AdaBoost [15] to class imbalance problems and showed it to be effective in increasing the AUC [16]. Chawla et al. [17] applied SMOTE before each Adaboost iteration while Seiffert et al. [18] used undersampling, both showing positive results. Liu et al. [19] proposed EasyEnsemble that builds multiple models using different subsamples of the majority class and then computes the sum of the posterior probability of the class label assigned by the base classifiers. use of clustering to partition the majority class and then build a model for each partition along with all the instances of the minority class. Experimental validation of clustering approaches to the imbalance problem, by Molinara [20], however, suggest that random partitioning is actually superior to clustering based methods. While ensemble models address the shortcomings of undersampling by resampling the data, and hence minimizing information loss, the models generated are larger and more complex as they consist of a set of models and, potentially, a weighting associated with the base models.

\section{ALGORITHMS}

In this paper we evaluate six methods for dealing with class imbalance in our data. Four of these methods are sampling-based, one is cost-based and one uses a rather novel genetic programming based approach.

\subsection{Sampling-based Methods}

Random Over Sampling (ROS): This method generates a sample of the minority class by sampling with replacement, effectively making multiple copies of the existing instances. An $\mathrm{r} \%$ oversample results in a data set consisting of all the majority class instances and $(1+r / 100) \times n$ minority class instances, where $n$ is the number of minority class instances within the original data set. In our experiments we varied the oversampling parameter $\mathrm{r}$ from $100 \%$ to $1500 \%$ in step sizes of $200 \%$.

Random Under Sampling (RUS): RUS reduces the number of majority class training instances by randomly removing instances belonging to the class from the training instances. The final number of instances used for training when using $\mathrm{r} \%$ under sampling is $r \times n / 100$, where $n$ is the number of majority class instances within the original data set. In our experiments we varied the value of $\mathrm{r}$ from $5 \%$ to $100 \%$ in steps of $5 \%$.

SMOTE: This is an implementation of Synthetic Minority Over-sampling TEchnique as proposed by Chawla et al [13]. It takes two parameters, the level of oversampling $r$ and the number of neighbours, $\mathrm{k}$, used for generating the synthetic instances. We experimented with values of $\mathrm{r}$ ranging from $100 \%$ to $1500 \%$ with step size of $200 \%$ and $\mathrm{k}$ taking integer values in the interval $[1,15]$.

Tomek: This is an implementation of Kubat and Matwin's [14] 


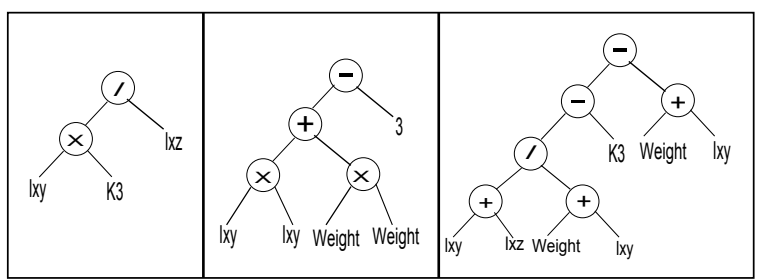

Figure 2: Example Chromosome

approach to one-sided selection.

\subsection{GP}

The Genetic Programming (GP) approach to evolutionary computing breeds a population of candidate programs to solve a problem [21]. These programs are typically represented as tree structures that are evolved over multiple iterations (generations). The initial population is generated at random while future generations are evolved through the measurement of fitness of the candidates programs and subsequently selecting candidates for reproduction with a probability proportional to their fitness. The candidate parent programs generate offsprings through the application of the crossover and mutation genetic operators.

We use a GP to generate a projection of the original data set to a feature space that is more amenable to learning a classification model in the presence of a skewed class distribution. Hence given a training data set, $D \subset \Re^{n}$, defined using a set of $n$ attributes $A$, labelled using one of two class labels $\left\{L_{1}, L_{2}\right\}$, we aim to learn a set of $k$ features, where each feature is a function of a subset of attributes in A and constants within some continuous range. Hence an individual (chromosome), in our case, consists of a set of $k$ programs, each representing a new dimension in the feature space. The function set, $F$, used to construct these features are the ordinary arithmetic functions $\left\{+,-,{ }^{*}, /\right\}$ where, as is common in GPs, ' $/$ ' is the protected division function that returns a value 1 when the denominator is 0 . The terminals (nodes within a program tree with no children) are either attributes or constants. An example chromosome is shown in Figure 2, representing a three-dimensional feature space defined by the features $\frac{I_{X Y} K_{3}}{I_{X Z}}, I_{X Y}^{2}+W e i g h t^{2}-3$ and $\frac{I_{X Y}+I_{X Z}}{\text { Weight }+I_{X Y}}-K_{3}-\left(\right.$ Weight $\left.+I_{X Y}\right)$.

To evaluate the fitness of a chromosome, a learning algorithm is applied to the data projected to the feature space using projections as defined by the chromosome. 10-fold cross validation is used to compute a composite confusion matrix. The confusion matrix is then used to compute the chromosomes' fitness defined as the area under the ROC curve (AUC).

The population is initialized using the Ramped half-andhalf method [22]. Chromosomes with an above average fitness within the current population are chosen to produce the next generation. This is acchieved through crossover and mutation. Subtree and gene crossover and subtree mutation are used to generate the new generation.

\subsection{Cost-Based Methods}

Cost-based methods assume the availability of a cost matrix defining the cost of misclassification of an instance belonging to class $C_{i}$ into class $C_{j}$. Class imbalance can be viewed as a misclassification cost minimization problem by assigning a higher cost to misclassification of a positive instance as a negative instance. MetaCost [23] is a popular method for dealing with cost minimization problems. Its attractiveness stems from the fact that it can be used with any base classifier. It works by estimating the posterior probability of the class labels given a training instance and then relabels the instance so as to minimize the expected misclassification cost. The final model is learnt from the relabelled training instance.

We experimented with a number of cost matricies. For the Olympic data set we evaluated a cost of missclassifying a positive instance as a negative one of 2 through to 10 in steps of 2. For the Soccer data that have a higher skew we experimented with values ranging from 5 to 55 in steps of 5 .

With each of the methods above, we used J48 (the implementation of C4.5 [24] in WEKA), JRip (the WEKA implementation of Ripper [25]), PART [26] and AdaBoost [15] to build models.

\section{RESULTS}

To find the optimal parameters for the individual sampling methods and estimate the expected quality of the model learnt, 10-fold cross validation was used to generate the confusion matrices for each of the four algorithms.

The cross validation was run ten times using different random seeds to account for the stochastic nature of the genetic algorithm and random sampling. The average AUC was used for selecting the best parameters for each model/sampling method.

Tables 2 and 3 summarize the results obtained. For each of the seven methods used to build models (four using sampling, 1 using the GP, 1 using MetaCost and 1 using a combination of sampling and ensemble learning), the precision and recall, using a threshold of 0.5 on the posterior probability of the class labels given an instance, estimated by the models learnt, area under the ROC curve (AUC) and the model complexity are shown. For each base algorithm (J48, Ripper and PART), we also compare the performance of the models with a base model (No Sampling) that does not apply any approach to address class imbalance.

The AUC gives us a summary of the model performance for all possible thresholds used on the posterior probability of the class labels to classify an instance as positive or negative. From the tables it is apparent that all methods for addressing the class imbalance with the exception of Random Oversampling (ROS) improve the AUC considerably over the base value (no sampling). On the whole, AdaBoost outperforms all other classifiers. The only exception being MetaCost when applied to the Soccer data with the base algorithm PART. Note however that there is no consensus as to which class imbalance method produces the best model when applied to AdaBoost,although SMOTE seems to perform better than the other approaches in general. With regard to the oversampling methods, SMOTE appears to outperform random oversampling. The latter appears to overfit both data sets and often performs worse than "No Sampling". When considering only classifiers consisting of a single model, the GP based approach using J48 performs best on the Olympic data set while MetaCost outperforms all classifiers on the Soccer data, including AdaBoost. Of the sampling based methods SMOTE and undersampling build models comparable in their AUC values and produce 
more accurate models than the GP or MetaCost when using Ripper on both data sets.

Model complexity represents the size of the obtained models. It is measured as the number of conditions in the model. In the case of trees, it is the number of nodes. In rules it is the number of conditions used. With respect to model complexity we can see that some of the best models are relatively small. This is important since, as we have mentioned, we intend to embed our classification model as JavaScript in the web page. The results for AdaBoost are not shown in the tables because the resulting model is an ensemble of ten base classifiers and hence would be overly complex for our purposes. The results of the PART method do not include model complexity due to WEKA not providing these through its API. We, however, expect PART models to show similar patterns to those of J48 and Ripper in that we would expect the GP and MetaCost to build smaller and more accurate model than the other methods studied in the paper. Random oversampling and SMOTE tend to build more complex models suggesting their susceptibility to overfitting.

With regard to Precision and Recall, the Olympic data set seems to represent an easier problem than the Soccer data set, perhaps because it is less imbalanced. Precision is in general quite low for the Soccer data (between 0.1 and 0.3 ) but higher for the Olympic data (0.3 to 0.53). Recall is also higher in general for the Olympic data set. Low values of precision are not very practical for the application we have in mind. An interest model with a precision of 0.2 will fire wrongly $80 \%$ of the time. We must note, however, that at deployment time, the models can be used with higher thresholds, which increases precision (but lowers recall). Figures 3 and 4 show the precision and recall curves for the best performing models, MetaCost and AdaBoost. As shown in Figure 3 for all base algorithms AdaBoost has the ability to deliver higher precision models than MetaCost. When using PART as the base algorithm, higher precision models are obtained by MetaCost when high recall is desirable. However, the difference in precision is rather small. Figure 4 shows the corresponding precision-recall curves for the Soccer data set. When using PART or Ripper as the base algorithm, MetaCost can learn models of higher precision when recall is in the range 0.2 to 0.6 in the case of PART and 0.1 to 0.4 in the case of Ripper. However, AdaBoost once again can provide higher precision models in general.

\section{CONCLUSION}

In this paper we have studied a large number of machine learning techniques for modeling user interest. We have used two data sets and assessed the techniques using a demanding experimental evaluation methodology. All these techniques other than random oversampling are adequate, in principle, for dealing with imbalanced classification problems, as is the case in user interest data sets. We conclude that a few techniques can be quite useful for dealing with these data sets. The most interesting compromise in terms of model complexity and AUC is MetaCost using PART as the base classification algorithm. AdaBoost yields higher AUC values but high complexity models. Therefore, our advice for this kind of user interest modeling application would be to use MetaCost and PART unless high precision at the cost of lower recall is desirable in which case it may be worth handling the additional complexity of AdaBoost models built

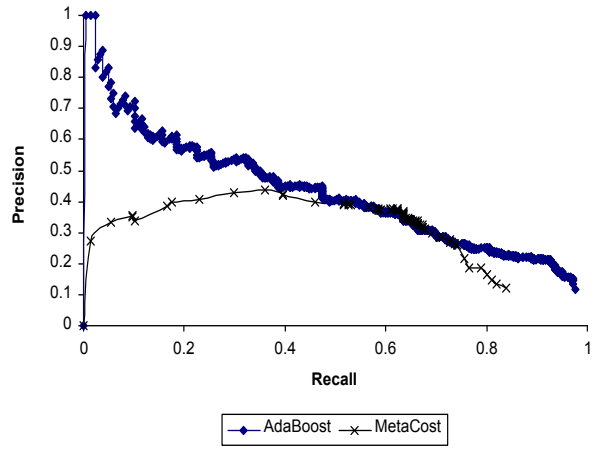

(a) J48

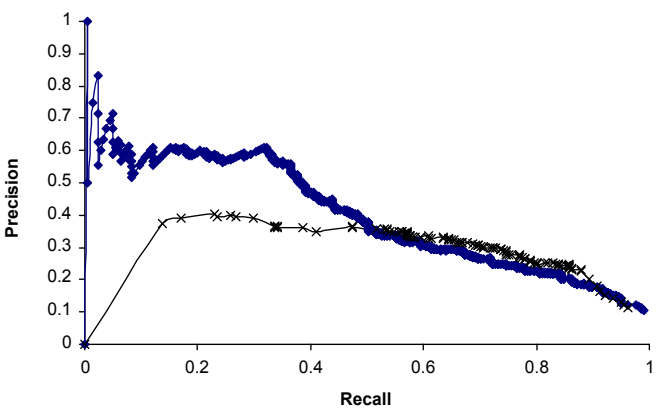

- AdaBoost $\star$ MetaCost

(b) PART

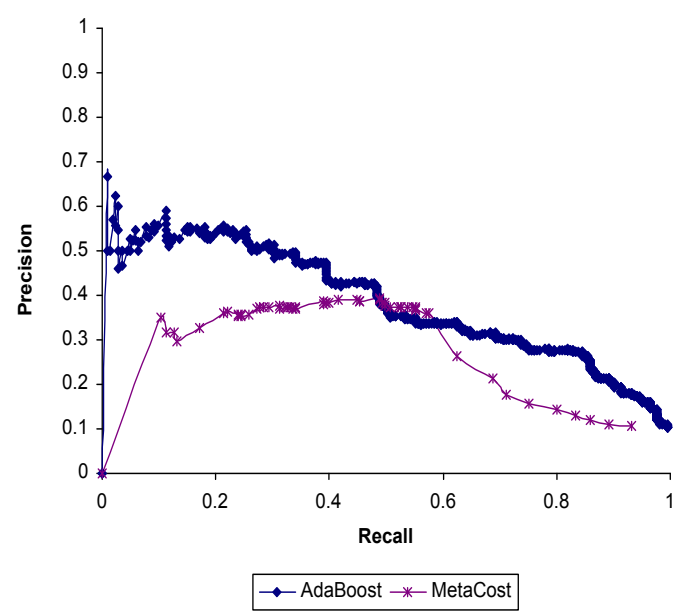

(c) Ripper

Figure 3: Recall-Precision Curves for select models for the Olympic Data Set 


\begin{tabular}{|c|c|c|c|c|c|}
\hline Algorithm & Parameters & Precision & Recall & $\mathrm{AUC}$ & $\begin{array}{l}\text { Model Com- } \\
\text { plexity }\end{array}$ \\
\hline \multirow{8}{*}{ J48 } & No Sampling & $0.516 \pm 0.031$ & $0.286 \pm 0.023$ & $0.74 \pm 0.012$ & $67.66 \pm 1.38$ \\
\hline & RUS $15 \%$ & $0.446 \pm 0.027$ & $0.456 \pm 0.022$ & $0.774 \pm 0.012$ & $43 \pm 3.79$ \\
\hline & ROS $1100 \%$ & $0.339 \pm 0.005$ & $0.432 \pm 0.01$ & $0.67 \pm 0.009$ & $255.38 \pm 2.61$ \\
\hline & SMOTE $100 \%$ N:14 & $0.42 \pm 0.014$ & $0.43 \pm 0.016$ & $0.77 \pm 0.008$ & $106.88 \pm 3.03$ \\
\hline & Tomek & $0.455 \pm 0.016$ & $0.445 \pm 0.03$ & $0.74 \pm 0.002$ & $75.74 \pm 1.34$ \\
\hline & GA Wrapper M:10 & $0.536 \pm 0.033$ & $0.259 \pm 0.015$ & $0.816 \pm 0.005$ & $20.76 \pm 1.77$ \\
\hline & MetaCost C: 5 M:10 & $0.378 \pm 0.009$ & $0.622 \pm 0.014$ & $0.802 \pm 0.003$ & $20.28 \pm 1.453$ \\
\hline & $\begin{array}{l}\text { AdaBoost SMOTE } 1300 \% \\
\mathrm{~N}: 13\end{array}$ & $0.391 \pm 0.012$ & $0.493 \pm 0.023$ & $0.834 \pm 0.008$ & - \\
\hline \multirow{8}{*}{ PART } & No Sampling & $0.423 \pm 0.017$ & $0.219 \pm 0.013$ & $0.745 \pm 0.014$ & - \\
\hline & RUS $25 \%$ & $0.408 \pm 0.009$ & $0.37 \pm 0.006$ & $0.771 \pm 0.009$ & - \\
\hline & ROS $100 \%$ & $0.357 \pm 0.022$ & $0.335 \pm 0.022$ & $0.714 \pm 0.008$ & - \\
\hline & SMOTE $1300 \%$ N:3 & $0.365 \pm 0.014$ & $0.516 \pm 0.006$ & $0.802 \pm 0.008$ & - \\
\hline & Tomek & $0.409 \pm 0.008$ & $0.383 \pm 0.017$ & $0.768 \pm 0.003$ & - \\
\hline & GA Wrapper & $0.469 \pm 0.025$ & $0.222 \pm 0.03$ & $0.805 \pm 0.009$ & - \\
\hline & MetaCost C: 7 M:4 & $0.306 \pm 0.006$ & $0.671 \pm 0.014$ & $0.809 \pm 0.009$ & - \\
\hline & AdaBoost Tomek & $0.424 \pm 0.012$ & $0.444 \pm 0.021$ & $0.831 \pm 0.002$ & - \\
\hline \multirow{8}{*}{ Ripper } & No Sampling & $0.443 \pm 0.015$ & $0.259 \pm 0.014$ & $0.618 \pm 0.009$ & $11.56 \pm 1.24$ \\
\hline & RUS $10 \%$ & $0.442 \pm 0.008$ & $0.413 \pm 0.015$ & $0.777 \pm 0.001$ & $4.51 \pm 0.019$ \\
\hline & ROS $500 \%$ & $0.331 \pm 0.01$ & $0.532 \pm 0.013$ & $0.717 \pm 0.009$ & $144.83 \pm 3.9$ \\
\hline & SMOTE $900 \%$ N:3 & $0.324 \pm 0.006$ & $0.547 \pm 0.011$ & $0.792 \pm 0.004$ & $94.22 \pm 2.06$ \\
\hline & Tomek & $0.448 \pm 0.013$ & $0.423 \pm 0.014$ & $0.697 \pm 0.006$ & $15.33 \pm 0.66$ \\
\hline & GA Wrapper & $0.317 \pm 0.017$ & $0.487 \pm 0.033$ & $0.65 \pm 0.099$ & $9.025 \pm 0.694$ \\
\hline & MetaCost C: 8 M:2 & $0.365 \pm 0.007$ & $0.56 \pm 0.012$ & $0.742 \pm 0.009$ & $32.89 \pm 2.826$ \\
\hline & AdaBoost RUS $20 \%$ & $0.413 \pm 0.013$ & $0.394 \pm 0.013$ & $0.837 \pm 0.004$ & - \\
\hline
\end{tabular}

Table 2: Summary of Results for Olympic Data

\begin{tabular}{|c|c|c|c|c|c|}
\hline Algorithm & Parameters & Precision & Recall & $\mathrm{AUC}$ & $\begin{array}{l}\text { Model Com- } \\
\text { plexity }\end{array}$ \\
\hline \multirow{8}{*}{ J48 } & No Sampling & 0.0 & 0.0 & $0.527 \pm 0.037$ & $1.82 \pm 0.55$ \\
\hline & RUS $30 \%$ & $0.025 \pm 0.049$ & $0.004 \pm 0.008$ & $0.6 \pm 0.003$ & $4.88 \pm 0.119$ \\
\hline & ROS $100 \%$ & $0.102 \pm 0.045$ & $0.111 \pm 0.007$ & $0.655 \pm 0.016$ & $53.48 \pm 2.75$ \\
\hline & SMOTE $100 \%$ N:7 & $0.131 \pm 0.058$ & $0.15 \pm 0.019$ & $0.744 \pm 0.015$ & $38.34 \pm 1.37$ \\
\hline & Tomek & $0.05 \pm 0.098$ & $0.02 \pm 0.004$ & $0.561 \pm 0.029$ & $3.72 \pm 0.955$ \\
\hline & GA Wrapper & $0.278 \pm 0.073$ & $0.082 \pm 0.027$ & $0.776 \pm 0.014$ & $13.32 \pm 1.12$ \\
\hline & MetaCost C:45 M:5 & $0.068 \pm 0.0003$ & $0.915 \pm 0.008$ & $0.775 \pm 0.006$ & $3.86 \pm 0.165$ \\
\hline & AdaBoost RUS $65 \%$ & $0.223 \pm 0.028$ & $0.147 \pm 0.023$ & $0.799 \pm 0.018$ & - \\
\hline \multirow{8}{*}{ PART } & No Sampling & $0.162 \pm 0.045$ & $0.058 \pm 0.016$ & $0.687 \pm 0.02$ & - \\
\hline & RUS $80 \%$ & $0.15 \pm 0.015$ & $0.065 \pm 0.006$ & $0.73 \pm 0.014$ & - \\
\hline & ROS $100 \%$ & $0.227 \pm 0.023$ & $0.193 \pm 0.025$ & $0.606 \pm 0.046$ & - \\
\hline & SMOTE $1300 \%$ N:5 & $0.127 \pm 0.009$ & $0.308 \pm 0.029$ & $0.722 \pm 0.032$ & - \\
\hline & Tomek & $0.145 \pm 0.02$ & $0.065 \pm 0.006$ & $0.708 \pm 0.021$ & - \\
\hline & GA Wrapper & $0.257 \pm 0.065$ & $0.074 \pm 0.023$ & $0.729 \pm 0.02$ & - \\
\hline & MetaCost C: 20 M:5 & $0.145 \pm 0.012$ & $0.584 \pm 0.028$ & $0.852 \pm 0.01$ & - \\
\hline & $\begin{array}{l}\text { AdaBoost SMOTE } 1300 \% \\
\text { N:6 }\end{array}$ & $0.191 \pm 0.016$ & $0.16 \pm 0.014$ & $0.807 \pm 0.02$ & - \\
\hline \multirow{8}{*}{ Ripper } & No Sampling & $0.297 \pm 0.096$ & $0.045 \pm 0.018$ & $0.52 \pm 0.012$ & $2.9 \pm 0.4$ \\
\hline & RUS $5 \%$ & $0.258 \pm 0.045$ & $0.074 \pm 0.032$ & $0.73 \pm 0.032$ & $3.36 \pm 0.325$ \\
\hline & ROS $1100 \%$ & $0.1512 \pm 0.015$ & $0.247 \pm 0.028$ & $0.621 \pm 0.013$ & $63.89 \pm 1.96$ \\
\hline & SMOTE $500 \%$ N:14 & $0.219 \pm 0.021$ & $0.35 \pm 0.042$ & $0.686 \pm 0.013$ & $39.63 \pm 1.40$ \\
\hline & Tomek & $0.214 \pm 0.047$ & $0.041 \pm 0.018$ & $0.528 \pm 0.014$ & $2.94 \pm 0.68$ \\
\hline & GA Wrapper & $0.31 \pm 0.046$ & $0.083 \pm 0.13$ & $0.544 \pm 0.011$ & $4.233 \pm 0.462$ \\
\hline & MetaCost C: 40 M:5 & $0.155 \pm 0.017$ & $0.27 \pm 0.031$ & $0.643 \pm 0.01$ & $13.17 \pm 0.779$ \\
\hline & $\begin{array}{l}\text { AdaBoost SMOTE } 1100 \% \\
\mathrm{~N}: 13\end{array}$ & $0.157 \pm 0.008$ & $0.152 \pm 0.006$ & $0.806 \pm 0.025$ & - \\
\hline
\end{tabular}

Table 3: Summary of Results for the Soccer Data 


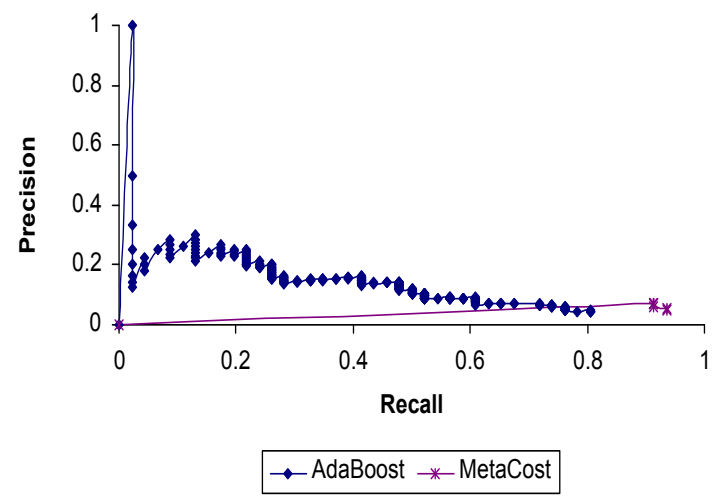

(a) $\mathrm{J} 48$

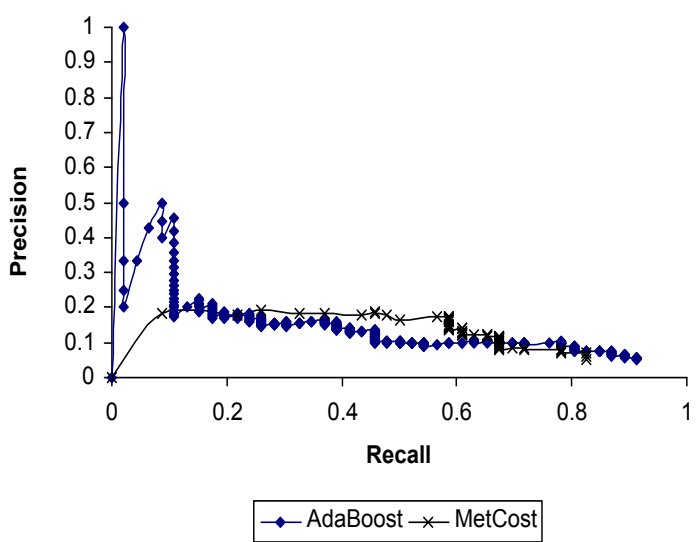

(b) PART

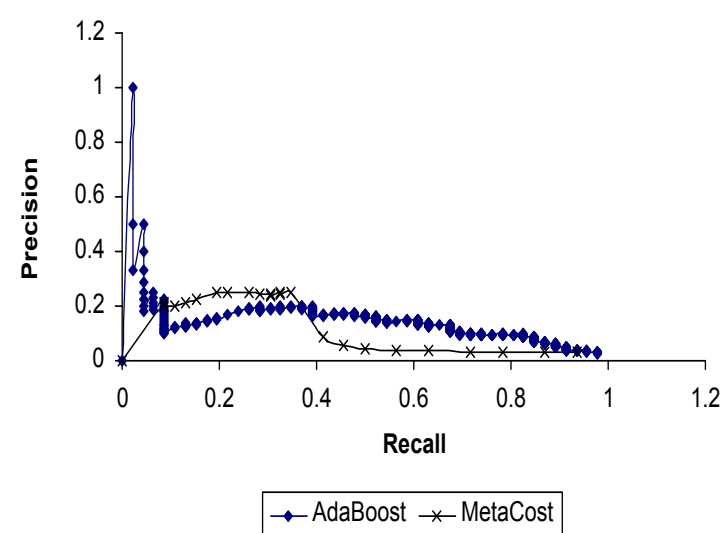

(c) Ripper

Figure 4: Recall-Precision Curves for select models for the Soccer Data Set using SMOTE. The accuracy of the AdaBoost based models seems fairly independent of the base classification method used.

\section{Acknowledgment}

Pest, SIBILA.

\section{REFERENCES}

[1] J. Huang and C. X. Ling, "Using auc and accuracy in evaluating learning algorithms - appendices," IEEE Trans. Knowl. Data Eng., vol. 17, no. 3, 2005.

[2] H. Lieberman, "Letizia: An agent that assists web browsing," in IJCAI (1), 1995, pp. 924-929.

[3] M. Claypool, P. Le, M. Waseda, and D. Brown, "Implicit interest indicators," in IUI, 2001, pp. 33-40.

[4] J. Goecks and J. W. Shavlik, "Learning users' interests by unobtrusively observing their normal behavior," in IUI, 2000, pp. 129-132.

[5] D. Hauger and L. V. Velsen, "Analyzing client-side interactions to determine reading behavior," in 17 th Workshop on adaptivity and user modeling in interactive systems (ABIS), 2009.

[6] K. Hofmann, C. Reed, and H. Holz, "Unobtrusive data collection for web-based social navigation," in Workshop on the Social Navigation and Community based Adaptation Technologies, 2006.

[7] T. F. Syeda-Mahmood and D. B. Ponceleon, "Learning video browsing behavior and its application in the generation of video previews," in $A C M$ Multimedia, 2001, pp. 119-128.

[8] Y. Hijikata, "Implicit user profiling for on demand relevance feedback," in IUI, J. Vanderdonckt, N. J. Nunes, and C. Rich, Eds. ACM, 2004, pp. 198-205.

[9] X. Yang, P. Xiang, and Y. Shi, "Finding user's interest blocks using significant implicit evidence for web browsing on small screen devices," World Wide Web, vol. 12, no. 2, pp. 213-234, 2009.

[10] D. Hauger, A. Paramythis, and S. Weibelzahl, "Your browser is watching you: Dynamically deducing user gaze from interaction data," in User Modelling, Adaptation and Personalization adjunct proceedings (posters), UMAP 2010, 2010.

[11] A. M. Jorge, J. P. Leal, and H. Dias, "Modeling user interest from client-side data with implicit labels," in under revision, 2011, pp. - .

[12] N. V. Chawla, D. A. Cieslak, L. O. Hall, and A. Joshi, "Automatically countering imbalance and its empirical relationship to cost," Data Min. Know. Disc., vol. 17, no. 2, pp. 225-252, 2008.

[13] N. V. Chawla, K. W. Bowyer, L. O. Hall, and W. P. Kegelmeyer, "Smote: Synthetic minority over-sampling technique," Journal of Art. Int. Research, vol. 16, pp. 321-357, 2002.

[14] M. Kubat and S. Matwin, "Addressing the curse of imbalanced training sets: One-sided selection," in In Proceedings of the Fourteenth International Conference on Machine Learning. Morgan Kaufmann, 1997, pp. 179-186.

[15] Y. Freund and R. E. Schapire, "Experiments with a new boosting algorithm," in In Proceedings of the 
Thirteenth International Conference on Machine Learning, 1996, pp. 148-156.

[16] C. Seiffert, T. M. Khoshgoftaar, J. V. Hulse, and A. Napolitano, "Building useful models from imbalanced data with sampling and boosting," in FLAIRS, 2008, pp. 306-311.

[17] N. V. Chawla, A. Lazarevic, L. O. Hall, and K. W. Bowyer, "Smoteboost: improving prediction of the minority class in boosting," in Proceedings of PKDD, 2003, pp. 107-119.

[18] C. Seiffert, T. M. Khoshgoftaar, J. V. Hulse, and A. Napolitano, "Rusboost: Improving classification performance when training data is skewed." in $I C P R$. IEEE, 2008, pp. 1-4.

[19] X.-Y. Liu, J. Wu, and Z.-H. Zhou, "Exploratory under-sampling for class-imbalance learning," in Proceedings of ICDM, 2006, pp. 965-969.

[20] M. Molinara, M. T. Ricamato, and F. Tortorella, "Facing imbalanced classes through aggregation of classifiers," in ICIAP '07: Proceedings of the 14th International Conference on Image Analysis and Processing. Washington, DC, USA: IEEE Computer Society, 2007, pp. 43-48.

[21] J. R. Koza, M. A. Keane, M. J. Streeter, W. Mydlowec, J. Yu, and G. Lanza, Genetic Programming IV: Routine Human-Competitive Machine Intelligence. Norwell, MA, USA: Kluwer Academic Publishers, 2003.

[22] S. Luke, "A survey and comparison of tree generation algorithms," in Proceedings of the Genetic and Evolutionary Computation Conference. Morgan Kaufmann, 2001, pp. 81-88.

[23] P. Domingos, "Metacost: A general method for making classifiers cost-sensitive," in In Proceedings of the Fifth International Conference on Knowledge Discovery and Data Mining. ACM Press, 1999, pp. 155-164.

[24] J. R. Quinlan, C4.5: Programs for Machine Learning. Morgan Kaufmann, 1992.

[25] W. W. Cohen, "Fast effective rule induction," in In Proceedings of the Twelfth International Conference on Machine Learning, 1995, pp. 115-123.

[26] E. Frank and I. H. Witten, "Generating accurate rule sets without global optimization," in Proceedings of the Fifteenth International Conference on Machine Learning, ser. ICML '98. San Francisco, CA, USA: Morgan Kaufmann Publishers Inc., 1998, pp. 144-151. [Online]. Available:

http://portal.acm.org/citation.cfm?id=645527.657305 